\title{
73. NUEVAS APORTACIONES A LA FLORA DE ANDALUCÍA: SIERRA DE GÁDOR (ALMERÍA)
}

\author{
Esther GIMÉNEZ LUQUE y Francisco GÓMEZ MERCADO
}

New floristic records for Andalusian: Sierra de Gádor (Almeria).

Palabras clave. Flora, corología, fitosociología, Almería, Andalucía.

Key words. Flora, corology, fitosociology, Almería, Andalusian.

Como resultado del estudio de flora y vegetación de la Sierra de Gádor (Almería), hemos herborizado abundante material del que entresacamos algunas primeras citas para esta sierra, señalando con un asterisco $(*)$ aquellas que son novedad para la provincia. Los pliegos testigo han sido depositados en el Herbario de la Universidad de Almería.

\section{Allium baeticum Boiss.}

ALMERÍA: Sierra de Gádor, El Florero, 30SWF1981, $1860 \mathrm{~m}$, en matorrales heliófilos, 15VI-1997, E. Giménez \& F. Gómez-Mercado. Herbario de la Universidad de Almería 1259.

\section{Althaea longiflora Boiss. \& Reut.}

ALMERÍA: Sierra de Gádor, Los Caleros, 30SWF1677, $1160 \mathrm{~m}$, en pastizales nitrófilos, 3-V1998, E. Giménez \& F. Gómez-Mercado. Herbario de la Universidad de Almería 1260. A pesar de existir citas de esta planta en Sierra de María (Cueto \& Blanca, 1998), según Paiva \& Nogueira (1993) no está citada en la provincia de Almería.

\section{Amelanchier ovalis Medik.}

ALMERÍA: Sierra de Gádor, Bco. de la Atalaya, 30SWF2081, $1400 \mathrm{~m}$, en espinales y matorrales caducifolios, 11-VI-1995, E. Giménez \& F. Gómez-Mercado. Herbario de la Universidad de Almería 1261.

Anthericum baeticum (Boiss.) Boiss.

ALMERÍA: Sierra de Gádor, próx. Cortijo Leitón, 30SWF2786, $1450 \mathrm{~m}$, en pastizales vivaces, 12-VI-1996, E. Giménez \& F. Gómez-Mercado.
Herbario de la Universidad de Almería 1262.

Bupleurum rigidum L. subsp. paniculatum (Brot.) H.Wolff.

ALMERÍA: Sierra de Gádor, Barranco de la Atalaya, 30SWF2284, $1650 \mathrm{~m}$, en herbazales del sotobosque del aceral-quejigal, 19-VI-1996, E. Giménez \& F. Gómez-Mercado. Herbario de la Universidad de Almería 1263.

\section{Buxus balearica Lam.}

ALMERÍA: Sierra de Gádor, Barranco de las Losas, 30SWF2793, en taludes rocosos umbríos y frescos, formando parte en estas situaciones del matorral subserial del encinar, 27-III-1998, E. Giménez \& F. Gómez-Mercado. Herbario de la Universidad de Almería 1264. Confirmamos su presencia en la Sierra de Gádor ya que con anterioridad se había citado en el Barranco Grande de Rágol y Barranco de Cacín (Sagredo, 1987) y para la Sierra de Gádor por Benedí (1997). A pesar de existir estas citas no había ningún pliego testigo que confirmara esta situación por lo que queda recogida como taxon dudoso en Almería por Cabezudo \& al. (1999).

\section{Chaenorrhinum robustum Loscos}

ALMERÍA: Sierra de Gádor, Hoya de los Gatos, 30SWF1783, $1950 \mathrm{~m}$, en sustratos pedregosos, 23-VI-1996, E. Giménez \& F. GómezMercado. Herbario de la Universidad de Almería 1265 .

Chaenorrhinum villosum (L.) Lange subsp. granatensis (Willk.) Valdés

ALMERÍA: Sierra de Gádor, Barranco de 
Martín, 30SWF2994, 550 m, rupícola, 13-VI-1996, E. Giménez \& F. Gómez-Mercado. Herbario de la Universidad de Almería 1266.

Epipactis parviflora (A. \& C. Nieschalk) E. Klein ALMERÍA: Sierra de Gádor, Fuente Alta, 30SWF1680, 1700 m, 31-V-1997, E. Giménez \& F. Gómez-Mercado. Herbario de la Universidad de Almería 1267.

Erodium saxatile Cav. ex Pau

ALMERÍA: Sierra de Gádor, Balsa de Barjalí, 30SWF1886, $1660 \mathrm{~m}$, rupícola y entre el matorral xeroacántico, 6-VI-1997, E. Giménez \& F. GómezMercado. Herbario de la Universidad de Almería 1268.

Erysimum incanum Kunze subsp. incanum

ALMERÍA: Sierra de Gádor, La Cruz, 30SWF2079, $1400 \mathrm{~m}$, en pastizales terofíticos algo nitrificados, 18-V-1996, E. Giménez \& F. GómezMercado. Herbario de la Universidad de Almería 1269.

Galium baeticum (Rouy) Ehrend. \& Krendl

ALMERÍA: Sierra de Gádor, Galería de Maldonado, 30SWF1083, $1850 \mathrm{~m}$, en matorrales xeroacánticos, 2-VII-1995, E. Giménez \& F. GómezMercado. Herbario de la Universidad de Almería 1270.

Galium nevadense Boiss. \& Reut.

ALMERÍA: Sierra de Gádor, Morrón del Francés, 30SWF1484, dolinas, $2200 \mathrm{~m}$, en pastizales de alta montaña, 16-VII-1996, E. Giménez \& F. Gómez-Mercado. Herbario de la Universidad de Almería 1271.

\section{Helianthemum neopiliferum Muñoz Garmendia \&}

C. Navarro

ALMERÍA: Sierra de Gádor, Balsón de las Hoyuelas, 30SWF1681, $1850 \mathrm{~m}$, en matorrales heliófilos, 23-VI-1996, E. Giménez \& F. GómezMercado. Herbario de la Universidad de Almería 1272.

*Helianthemum viscidulum Boiss. subsp. raynaudii (A. Ortega, Romero García \& C. Morales) G. López

ALMERÍA: Sierra de Gádor, Morrón del
Francés, 30SWF1484, $2200 \mathrm{~m}$, tomillares dolomíticos, 8-VII-1997, E. Giménez \& F. GómezMercado. Herbario de la Universidad de Almería 1273. Según López González (1993) es endémico de las proximidades del puerto de la Mora. Con esta nueva cita, ampliamos su área de distribución hasta la Sierra de Gádor.

Hormathophylla cadevalliana (Pau) T.R. Dudley ALMERÍA: Sierra de Gádor, Tajo de la Parra, 30SWF1289, $1600 \mathrm{~m}$, en comunidades rupícolas, 21-VI-1996, E. Giménez \& F. Gómez-Mercado. Herbario de la Universidad de Almería 1274.

*Iberis carnosa Willd. subsp. hegelmaieri (Willk.) Moreno

ALMERÍA: Sierra de Gádor, Barjalí, 30SWF1990, $1700 \mathrm{~m}$, en matorrales heliófilos, 6VI-1997, E. Giménez \& F. Gómez-Mercado. Herbario de la Universidad de Almería 1275. Endemismo de las sierras del levante español, ampliamos su área con esta cita a la provincia de Almería.

Iberis saxatilis L. subsp. cinerea (Poir.) Font Quer ALMERÍA: Sierra de Gádor, Cerro del Pastor, 30SWF2279, $1550 \mathrm{~m}$, en suelos pedregosos sobre sustratos calizos, 9-VI-1996, E. Giménez \& F. Gómez-Mercado. Herbario de la Universidad de Almería 1276.

\section{Lemna gibba L.}

ALMERÍA: Sierra de Gádor, La Alquería, 30SWF0070, $40 \mathrm{~m}$, en aguas estancadas eutrofizadas, 7-V-1999, E. Giménez \& F. GómezMercado. Herbario de la Universidad de Almería 1277.

\section{Lepidium heterophyllum Benth}

ALMERÍA: Sierra de Gádor, Balsa del Florero, 30SWF1981, $1850 \mathrm{~m}$, en comunidades pascícolas de alta montaña, 15-VI-1997, E. Giménez \& F. Gómez-Mercado. Herbario de la Universidad de Almería 1278. A pesar de existir una cita de este taxon para la Sierra de Gádor: Cabecera del Bco. del Pilar, WF1786, 1600 m (Pallarés, 1988), en Flora Ibérica (Hernández Bermejo \& Clemente, 1993) se cita en la provincia de Almería únicamente en Sierra Nevada. 


\section{Lepidium petrophilum Coss.}

ALMERÍA: Sierra de Gádor, Hoya de los Gatos, 30SWF1783, 1950 m, comunidades pisoteadas por el ganado próximas a balsas de alta montaña (Polygono-Poetea annuae), 23-VI-1996, E. Giménez \& F. Gómez-Mercado. Herbario de la Universidad de Almería 1279. Endemismo de las sierras de Alcaraz, Segura, Cazorla, Mágina y Sierra Nevada, ampliamos su área de distribución a la Sierra de Gádor.

Melica ciliata L. subsp. monticola (Prokudin) Tzevelev

ALMERÍA: Sierra de Gádor, Morrones, 30SWF1484, $2200 \mathrm{~m}$, en pastizales de alta montaña, 16-VII-1996, E. Giménez \& F. Gómez-Mercado. Herbario de la Universidad de Almería 1280. Segunda cita peninsular, la primera es de la Sierra de los Filabres, en "la Alfaguara, Sierro, WG5425 a 1400 m” (Pallarés, 1994).

Moehringia intricata Willk. subsp. intricata

ALMERÍA: Sierra de Gádor, Piedra del Lastonar, 30SWF2179, $1550 \mathrm{~m}$, en grietas de rocas calizas húmedas y umbrosas, 9-VI-1996, E. Giménez \& F. Gómez-Mercado, Herbario de la Universidad de Almería 1281. Segunda cita para la Sierra de Gádor, Montserrat (1988).

\section{*Muscari atlanticum Boiss. \& Reut.}

ALMERÍA: Sierra de Gádor, Rambla del Boquerón, 30SWF0977, $400 \mathrm{~m}$, en pastizales pisoteados, 1-III-1996, E. Giménez \& F. GómezMercado. Herbario de la Universidad de Almería 1282.

Onosma tricerosperma Lag. subsp. granatensis (Debeaux \& Degen) Stroh

ALMERÍA: Sierra de Gádor, Bco. de la Atalaya, 30SWF2081, $1700 \mathrm{~m}$, entre el matorral heliófilo, 19-VI-1996, 5-VII-1999, E. Giménez \& F. Gómez-Mercado. Herbario de la Universidad de Almería 1283.

\section{Phillyrea angustifolia $\mathrm{L}$.}

ALMERÍA: Sierra de Gádor, Bco. de la Fuente, 30SWF3582, $750 \mathrm{~m}$, en bosquetes esclerófilos, 7VI-1996, E. Giménez \& F. Gómez-Mercado. Herbario de la Universidad de Almería 1284.
Pistacia $\times$ saportae Burnat $(P$. lentiscus $\times P$. terebinthus)

ALMERÍA: Sierra de Gádor, Barranco de las Losas, 30SWF2792, 900 m, 7-V-1998, E. Giménez \& F. Gómez-Mercado. Herbario de la Universidad de Almería 1285.

Rhamnus saxatilis Jacq. subsp. saxatilis

ALMERÍA: Sierra de Gádor, Nuevo Mundo, 30SWF1882, $2080 \mathrm{~m}$, entre espinales caducifolios, 16-VI-1996, E. Giménez \& F. Gómez-Mercado. Herbario de la Universidad de Almería 1286.

Rumex papillaris Boiss. \& Reut.

ALMERÍA: Sierra de Gádor, Llano de la Chanata, 30SWF2787, $1400 \mathrm{~m}$, prados nitrificados, 23-V-1998, E. Giménez \& F. Gómez-Mercado, Herbario de la Universidad de Almería 1302.

Rumex pulcher L. subsp. woodsii (De Not.) Arcangeli

ALMERÍA: Sierra de Gádor, Fuente Plomeras, 30SWF1087, $1630 \mathrm{~m}$, borde de carril, 26-VI-1996, E. Giménez \& F. Gómez-Mercado. Herbario de la Universidad de Almería 1287.

Sorbus aria (L.) Crantz

ALMERÍA: Sierra de Gádor, Bco, de la Atalaya, 30SWF2081, $1400 \mathrm{~m}$, en bosquetes caducifolios sobre sustratos calizos, 11-VI-1995, E. Giménez \& F. Gómez-Mercado. Herbario de la Universidad de Almería 1288. No existía cita de su presencia en la Sierra de Gádor por lo que no ha sido recogida por Herrera \& al. (2000).

Stachys circinata L'Hér

ALMERÍA: Sierra de Gádor, Fuente Victoria, 30SWF1193, $850 \mathrm{~m}$, en herbazales con cierta humedad edáfica, E. Giménez \& F. Gómez-Mercado. Herbario de la Universidad de Almería 1289.

Veronica tenuifolia Asso subsp. fontqueri (Pau) Mart. Ortega \& E. Rico

ALMERÍA: Sierra de Gádor, El Florero, 30SWF1981, $1860 \mathrm{~m}$, matorrales heliófilos de alta montaña (Xeroacantho-Erinaceion), 15-VI-1997, E. Giménez \& F. Gómez-Mercado. Herbario de la Universidad de Almería 1290. Este taxon fue descrito por Pau (1922) con categoría específica 
para la Sierra de Gádor, posteriormente fue encontrada por Gros, Losa y Rivas en la Balsa del Sabinal (Sierra de Gádor, 1800 m.) (Sagredo, 1987). Actualmente, Martínez Ortega \& Rico (2000) le asignan al taxon categoría subespecífica. Pérez Latorre \& al. (1998), confirman la presencia de este taxon en la Serranía de Ronda considerandolo endemismo rondeño y gadorense. En la Sierra de Gádor también se ha citado Veronica austriaca L. en diversos puntos: Sierra de Gádor, sobre Castala (1700 m.), Llano del Sabinal (1800 m.), La Chanata, El Calabrial (Sagredo, 1987). Sierra de Gádor, Fondón, Llanos de Boliches, WF1786, $1650 \mathrm{~m}$ (Valdés \& Talavera, 1991), en opinión de Martínez Ortega (1998), todas estas citas han de referise al endemismo Veronica tenuifolia subsp. fontqueri, único representante del grupo en la Sierra de Gádor.

\section{BIBLIOGRAFÍA}

BENEDí, C. -1997- Buxus L. en Castroviejo, S. et al. (éds.). Flora Ibérica VIII: HaloragaceaeEuphorbiaceae. Real Jardín Botánico, CSIC. Madrid.

CABEZudo, B., A.V. PÉREZ LATORRE, D. NAVAS y Y. GIL -1999- Buxus balearica Lam. en Blanca et al. (eds.). Libro Rojo de la Flora Silvestre Amenazada de Andalucía. Tomo I: Especies en Peligro de Extinción. Consejería de Medio Ambiente. Junta de Andalucía.

CUETO, M. y G. BLANCA -1998- Flora del Parque Natural Sierra de María-Los Vélez. Ed. SAHN. Almería.

HERNÁNDEZ BERMEJO, J.E. y M. CLEMENTE 1993- Lepidium L. en Castroviejo, S. et al. (éds.). Flora Ibérica IV: CruciferaeMonotropaceae. Real Jardín Botánico, CSIC. Madrid.

HERRERA, C.M., A. MANZANEDA, A. BENAVENTE, P. LUQUE y P. JORDANO 2000- Sorbus aria (L.) Crantz en Blanca et al. (eds.). Libro rojo de la Flora Silvestre Amenazada de Andalucía. Tomo II: Especies Vulnerables. Consejería de Medio Ambiente. Junta de Andalucía.

LÓPEZ GONZÁLEZ, G. -1993- Helianthemum Mill. en Castroviejo, S. et al. (éds.). Flora Ibérica III: Plumbaginaceae (partim)-
Capparaceae. Real Jardín Botánico, CSIC. Madrid.

MARTÍNEZ ORTEGA, M.M. -1998-Revisión taxonómica de Veronica sect. Veronica L. y sect. Veronicastrum W.D.J. Koch en el Mediterráneo Occidental. Tesis Doctoral. Universidad de Salamanca.

MARTÍNEZ ORTEGA, M.M. y E. RICO -2000Algunas tipificaciones y combinaciones nuevas en las Veronica L. (Scrophulariaceae) perennes. Anales Jardín Bot. Madrid, 57(2):477-479.

MONTSERRAT, J.M. -1988- Morfología de las semillas de Moehringia gr. intricata (Caryophyllaceae). Lagascalia 15 (extra):195203.

PAIVA, J. e I. NOGUEIRA - 1993- Althaea L. en Castroviejo, S. et al. (éds.). Flora Ibérica III: Plumbaginaceae (partim)-Capparaceae. Real Jardín Botánico, CSIC. Madrid.

PALLARÉS, A. -1988- Addenda a la Flora de Almería. Boletín del IEA 8:117-160.

PALLARÉS, A. -1994- La familia de las gramíneas en Almería. Cuadernos Monográficos, $\mathrm{n}^{\circ} 27$. IEA. Diputación de Almería.

PAU, C. -1922- Las herborizaciones del Sr. Gros por la región almeriense. Buttl. Inst. Catalan Hist. Nat. 22:30-33.

PÉREZ LATORRE, A.V., P. NAVAS, D. NAVAS, Y. GIL y B. CABEZUDO -1998- Datos sobre la flora y vegetación de la Serranía de Ronda (Málaga, España). Acta Bot. Malacitana 23:149191.

SAGREDO, R. -1987- Flora de Almería. Plantas vasculares de la provincia. IEA. Almería.

VALDÉS, B. y S. TALAVERA -1991- Check list of the vascular plants collected during Iter Mediterraneum I. Bocconea 1:43-292.

Aceptado para su publicación en septiembre de 2001

Dirección de los autores. Departamento de Biología Vegetal y Ecología. Escuela Politécnica Superior. Universidad de Almería. E-04120 Almería. 